\title{
Role of Chemical Structure of Support in Enhancing the Catalytic Activity of a Single Atom Catalyst Toward NRR: A Computational Study
}

\author{
Thillai Govindaraja Senthamaraikannan ${ }^{1,2 *}$, Selvaraj Kaliaperumal ${ }^{2}$ and \\ Sailaja Krishnamurty ${ }^{3 *}$
}

${ }^{1}$ Department of Environmental Engineering, Chungbuk National University, Cheongju, Korea, ${ }^{2}$ Nano and Computational Material Lab, Catalysis Division, CSIR-National Chemical Laboratory, Pune, India, ${ }^{3}$ Physical Chemistry Division, CSIR-National Chemical Laboratory, Pune, India

OPEN ACCESS

Edited by: Lalith Perera

National Institute of Environmental Health Sciences (NIEHS),

United States

Reviewed by: Michael Springborg, Saarland University, Germany Debdutta Chakraborty, KU Leuven, Belgium

*Correspondence: Sailaja Krishnamurty k.sailaja@ncl.res.in

Thillai Govindaraja Senthamaraikannan thillaincı@gmail.com

Specialty section: This article was submitted to Theoretical and Computational Chemistry,

a section of the journal

Frontiers in Chemistry

Received: 30 June 2021 Accepted: 12 August 2021 Published: 08 September 2021

Citation:

Senthamaraikannan $T G$, Kaliaperumal $S$ and Krishnamurty $S$ (2021) Role of Chemical Structure of Support in Enhancing the Catalytic Activity of a Single Atom Catalyst Toward NRR: A Computational Study.

Front. Chem. 9:733422.

doi: $10.3389 /$ fchem.2021.733422
Using the periodic density functional theory-based methodology, we propose a potential catalytic system for dinitrogen activation, viz., single metal atoms (Mo, Fe, and V) supported on graphene-based sheets. Graphene-based sheets show an excellent potential toward the anchoring of single atoms on them (Mo, Fe, and V) with adsorption energies ranging between 1.048 and $10.893 \mathrm{eV}$. Factors such as defects and BN doping are noted to enhance the adsorption energies of single metal atoms on the support. The adsorption of a dinitrogen molecule on metal atom-anchored graphenebased supports is seen to be highly favorable, ranging between 0.620 and $2.278 \mathrm{eV}$. The adsorption is driven through a direct hybridization between the $d$ orbitals of the metal atom (Mo, Fe, and $\mathrm{V}$ ) on the support and the $p$ orbital of the molecular nitrogen. Noticeably, BNdoped graphene supporting a single metal atom ( $\mathrm{Mo}, \mathrm{Fe}$, and $\mathrm{V}$ ) activates the $\mathrm{N}_{2}$ molecule with a red shift in the $\mathrm{N}-\mathrm{N}$ stretching frequency $\left(1,597 \mathrm{~cm}^{-1}\right.$ as compared to $2,330 \mathrm{~cm}^{-1}$ in the free $\mathrm{N}_{2}$ molecule). This red shift is corroborated by an increase in the $\mathrm{N}-\mathrm{N}$ bond length (1.23 $\AA$ from $1.09 \AA$ ) and charge transfer to an $N_{2}$ molecule from the catalyst.

Keywords: $\mathrm{N}_{2}$ activation, single metal atom, pristine graphene, defective graphene, BN-functionalized graphene

\section{INTRODUCTION}

Ammonia is an important chemical substance for the agriculture, pharmaceuticals, and chemical industries. Natural and synthetic $\mathrm{N}_{2}$ fixation is necessary for the existence of all forms of life on Earth. Though the availability of dinitrogen $\left(\mathrm{N}_{2}\right)$ is abundant in air, it requires high energy for fixation and activation owing to its existence of inert triple bonds between nitrogen atoms. Currently, the wellknown Haber-Bosch process invented more than a century ago is used for converting dinitrogen $\left(\mathrm{N}_{2}\right)$ in the atmosphere into $\mathrm{NH}_{3}$ in the presence of the iron catalyst at an extreme temperature $\left(500^{\circ} \mathrm{C}\right)$ and pressure $(200 \mathrm{~atm})$ (Fryzuk and Johnson, 2000). The energy- and carbon-intensive Haber-Bosch process consumes 1-2\% global energy and, in addition, produces $3 \%$ of global $\mathrm{CO}_{2}$ emission (Cherkasov et al., 2015). Nevertheless, $\mathrm{N}_{2}$ fixation can occur readily under mild conditions by nitrogenase mechanism, the enzyme secreted from very few prokaryotic organisms (Kim and Rees, 1992; Sellmann and Sutter, 1997; Einsle et al., 2002). Researchers have demonstrated the occurrence of biological $\mathrm{N}_{2}$ fixation under reasonable or mild conditions in the presence of nitrogenase enzymes, most preferably at the active sites that are rich in $\mathrm{Fe}$ and $\mathrm{S}$ and also 


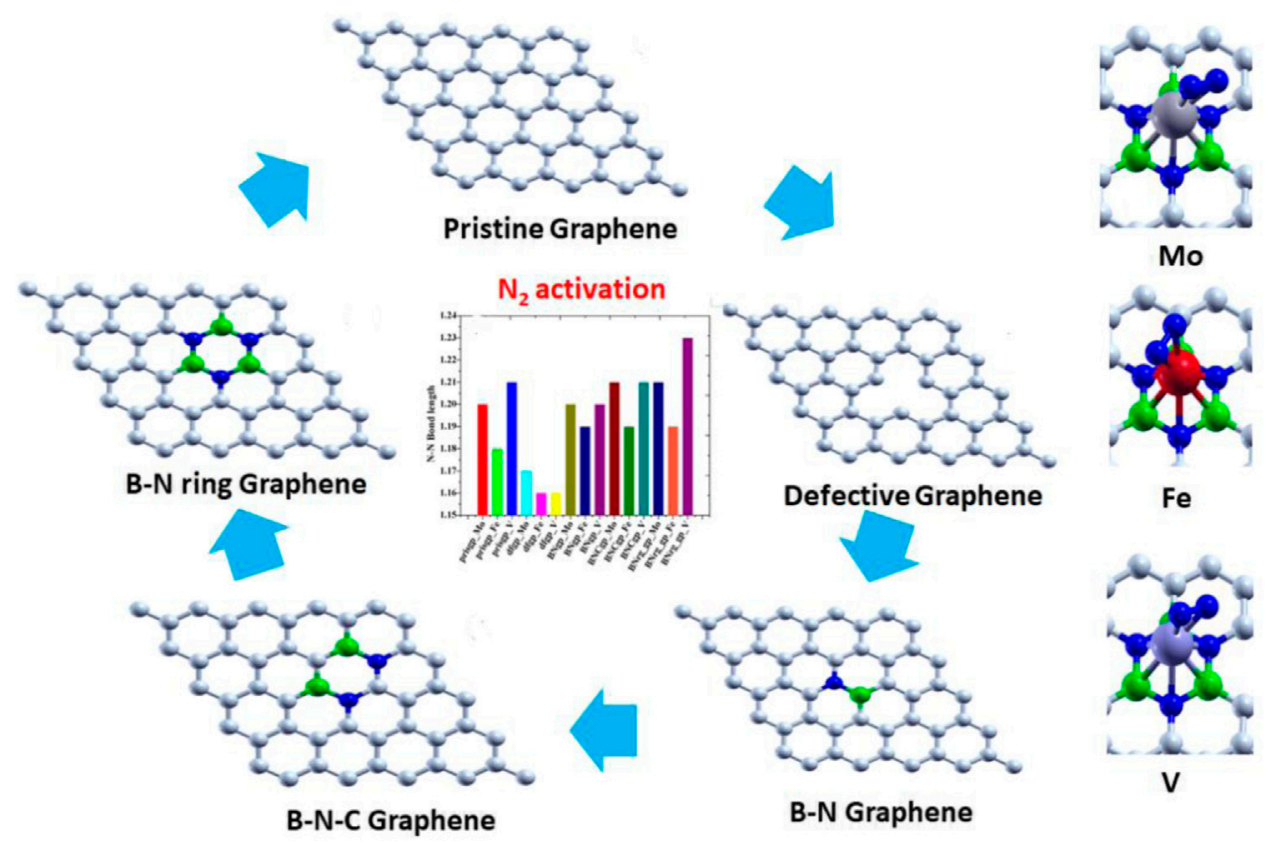

Graphical Abstract | XXX

additionally contain Mo or V atoms (Dance, 2008; Stüeken et al., 2015; Tanabe and Nishibayashi, 2016), yet the through kinetics are still disputed. Consequently, exploring an efficient $\mathrm{N}_{2}$ reduction catalyst in ammonia synthesis is the main challenge for the organo-metallic researchers. Naturally, $\mathrm{N}_{2}$ fixation and activation require a potential catalytic active center to promote nitrogen reduction reaction, via electrons overlapping between the $\sigma$ bond of $\mathrm{N}_{2}$ and the $\mathrm{d}$ orbital of the metal center, and the occupied $d$ orbital overlaps with the empty $\pi^{*}$ bond of $\mathrm{N}_{2}$, resulting in the activation of $\mathrm{N}_{2}$ by a $\pi$ bond back-donation mechanism.

On accounting for the quantum confinement of electrons, metal clusters are widely explored as catalysts. Using experimental and theoretical strategies, researchers have explored $\mathrm{N}_{2}$ activation on potential inorganic metal clusters (Seh et al., 2017; Liu et al., 2018; Wang et al., 2018). Significantly, Kerpal et al. (2013) have evaluated dinitrogen $\left(\mathrm{N}_{2}\right)$ activation using infrared multiphoton dissociation (IRMPD) on neutral Ru clusters. Similarly, Roy et al. (2009) have noticed the red-shifted $\mathrm{N}-\mathrm{N}$ bond stretching frequency around $810 \mathrm{~cm}^{-1}$ on solid $\mathrm{Li}_{\mathrm{n}}(2<\mathrm{n}<8)$ clusters, particularly the $\mathrm{Li}_{8}$ metal cluster showing an exothermic trend in splitting the $\mathrm{N}-\mathrm{N}$ bond completely. In the midst of metal clusters for evaluating $\mathrm{N}_{2}$ activation reaction, Al clusters play a remarkable role. Previously, Jarrold et al. observed low energy barriers for $\mathrm{N}_{2}$ activation on $\mathrm{Al}_{44}$ and $\mathrm{Al}_{100}$ clusters at high temperatures using concerted experimental and theoretical techniques (Cao et al., 2010). Similarly, in another previous report by this group, $\mathrm{N}_{2}$ activation potential was observed to be dependent on the phase and structure of the metal cluster (Cao et al., 2009).
During the course of $\mathrm{N}_{2}$ activation mechanism, conformations with high energy display low energy potential toward the activation of the $\mathrm{N}_{2}$ molecule (Kulkarni et al., 2011). Nevertheless, excited state conformations are meta-stable in nature and are notably present only at some characteristic finite temperatures. Hence, there is an obvious demand for more reliable and stable ground state conformations for $\mathrm{N}_{2}$ activation. Consequently, heteroatoms such as silicon and phosphorus doped on aluminum clusters appear to be a possible alternative and have better activation than their pristine aluminum clusters (Das et al., 2014).

Moreover, an alternative and experimentally supported route is to enhance the activity of metal-based catalysts by anchoring metal centers on 2D material supports such as graphene and $\mathrm{BN}$, which offers a substantial support to the metal centers to adsorb and activate the $\mathrm{N}_{2}$ molecule. Moreover, specific activity per metal atom increases by downsizing the metals from nanoparticles to nanocrystals or hetero-nano framework (Yang et al., 2013; Chen et al., 2014). Single atom catalysts (SACs) have gained more attention in downsizing metals considerably and exhibit the potential of welldispersed active single atom sites available for atomic utilization (Qiao et al., 2011). Based on these circumstances, SACs exhibiting unique activity with high density of active sites supported on 2D materials can make use of electron sharing for the activation of the inert dinitrogen molecule. A single transition-metal atom or atom clusters supported on $\mathrm{N}$-doped graphene show good nitrogen reduction reaction (NRR) activity (Choi et al., 2015; Li et al., 2016; Fajardo and Peters, 2017; Fei et al., 2018; Yan et al., 2019). Systems such 


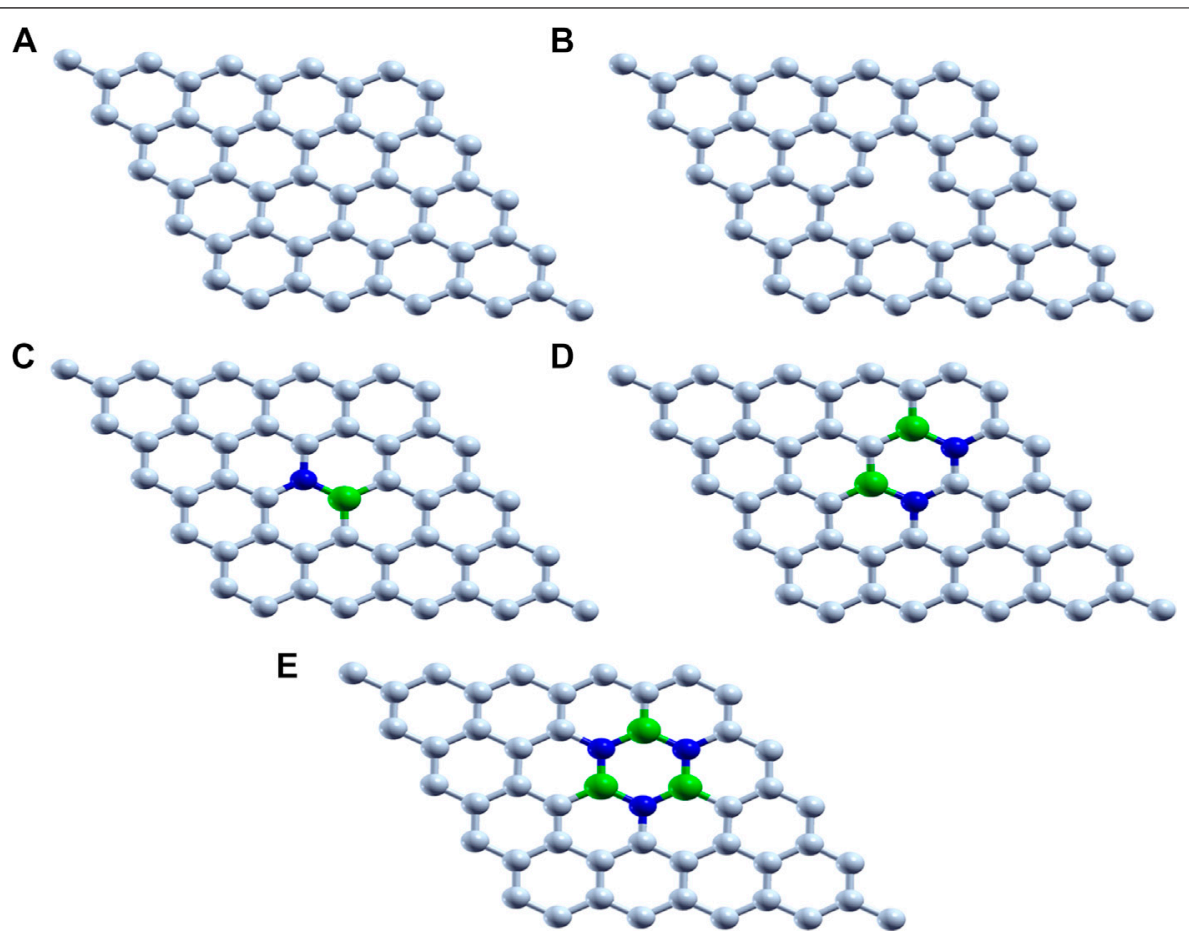

FIGURE 1 | Optimized structure of (A) pristine graphene, (B) defective graphene, (C) BN-doped graphene, (D) BNC-ring graphene, and (E) BN-ring graphene (C, white; B, green; and N, blue).

as $\mathrm{BiOBr}$ nanosheets, boron anti-sites on $\mathrm{BN}$ nanotubes, and Mo-doped boron nitride $(\mathrm{BN})$ have also been reported to have high $\mathrm{N}_{2}$ fixation potential (Li et al., 2015; Kumar and Subramanian, 2017; Zhao and Chen, 2017; Légaré et al., 2018).

In the midst of $2 \mathrm{D}$ materials, graphene-based supports attract enormous attention in numerous reactions such as water splitting, Guo et al. (2018), and hydrogen evolution reaction (HER) Ouyang et al. (2018). Few experimental groups reported $\mathrm{N}_{2}$ fixation using a graphene-based catalytic support (Jeon et al., 2013; Lu et al., 2016; Yan et al., 2018). Several computational investigations have also been explored using graphene-based nanomaterials for $\mathrm{N}_{2}$ fixation to compare with the experimental findings. Le et al. reported that the $\mathrm{Mo} /$ $\mathrm{N}$-doped graphene-based support dissociates the $\mathrm{N}_{2}$ molecule using the density functional theory (DFT) methodology (Le et al., 2014). In a similar approach, Li et al. observed an $\mathrm{N}_{2}$ molecule activation to nearly $2.5 \AA$ by fixing the $\mathrm{FeN}_{3}$ molecule on a graphene support, in which nitrogen atoms are used as anchoring elements, while iron does the activation job in the $\mathrm{FeN}_{3}$ molecule (Li et al., 2016). Kumar et al. (2016) reported $\mathrm{N}_{2}$ activation using aluminum clusters doped on the BN-doped graphene support. The rare ability of certain transition complexes to bind to $\mathrm{N}_{2}$, which is attributed to their advantageous combination of unoccupied and occupied $\mathrm{d}$-orbitals that have appropriate energy and symmetry to synergistically accept/back-donate electron density from/ to $\mathrm{N}_{2}$, can thus be contrived by giving the appropriate environment to a p-block element. In short, activation of $\mathrm{N}_{2}$ is performed by exploiting the electron reservoir property of $2 \mathrm{D}$ graphene-based materials. Recently, in our previous investigations, we identified the most active and recyclable SAC/B-graphene composite as the catalyst for NRR activity (Maibam et al., 2019; Maibam and Krishnamurty, 2021). In the present work, using the density functional theory (DFT)-based methodology, we evaluate the possible dinitrogen activation by single metal atoms (Mo, Fe, and V) supported on graphenebased systems such as pristine graphene, defective graphene, $\mathrm{BN}$ doped graphene, $\mathrm{BNC}$-ring graphene, and $\mathrm{BN}$-ring graphene as support materials.

\section{Computational Details}

We use the Vienna Ab Initio Simulation Package (VASP) (Kresse and Furthmller, 1996) with the PBE functional (Perdew et al., 1996) to perform all the first-principles calculations in the present work. The projected augmented wave (PAW) (Blöchl, 1994) method is employed using an energy cutoff of $520 \mathrm{eV}$ to describe the plane wave basis set. The two-dimensional graphene sheets are simulated using periodic boundary conditions. To avoid the interactions between the different nearest neighboring layers, a vacuum space of $20 \AA$ is created along the $Z$-direction. The $5 \times 5$ supercell with 50 atoms is used as the graphene surface model, and the optimized $\mathrm{C}-\mathrm{C}$ bond length in the graphene sheet is $1.42 \AA$.

Pristine graphene, defective graphene, $\mathrm{BN}$-doped graphene, $\mathrm{BNC}$-ring graphene, and $\mathrm{BN}$-ring graphene are designed surface 


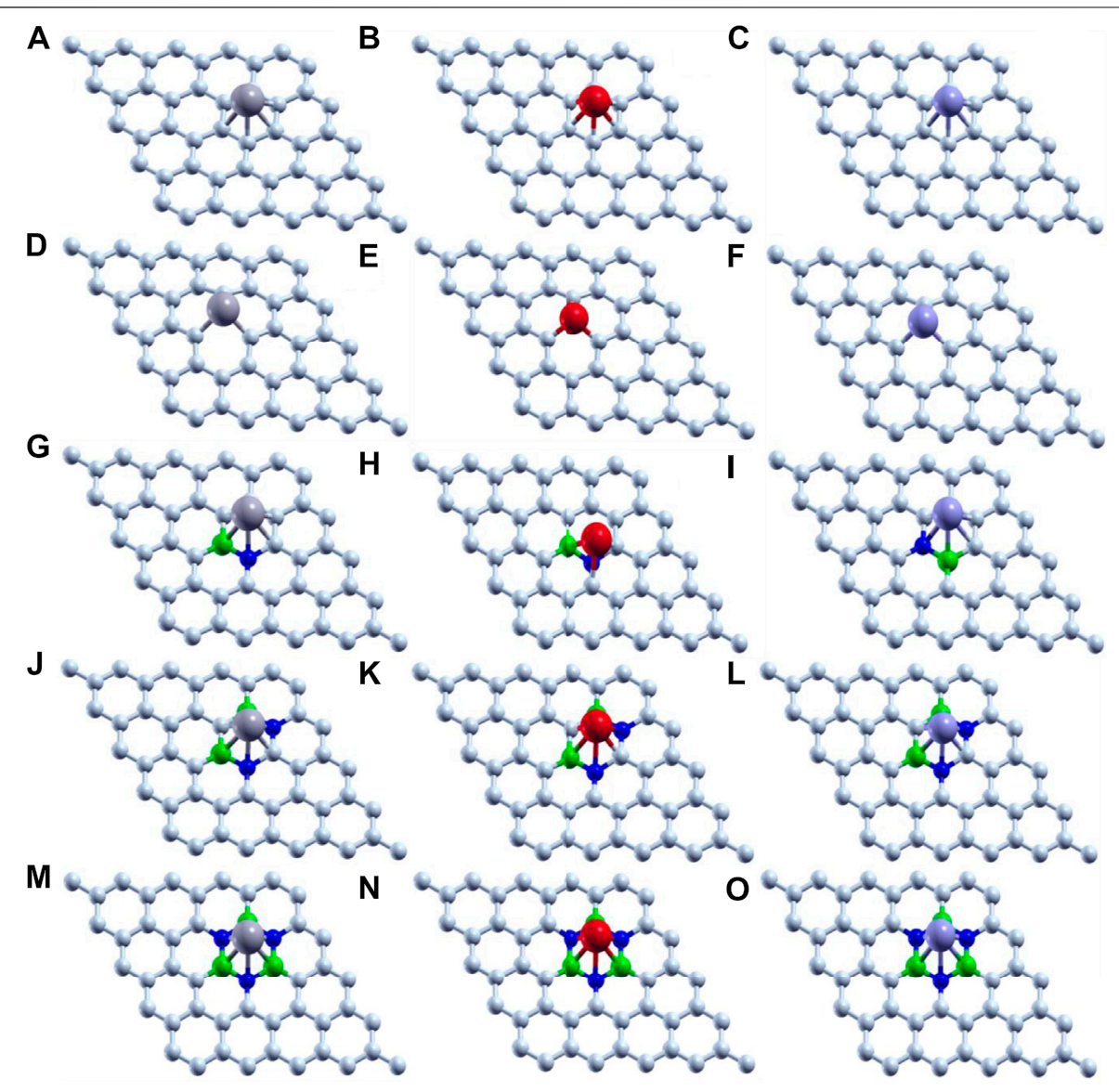

FIGURE 2 | Optimized structure of adsorption of Mo (gray), Fe (red), and V (purple) on (A-C) pristine graphene, (D-F) defective graphene, (G-I) BN-doped graphene, (J-L) BNC-ring graphene, and (M-O) BN-ring graphene.

supports, and the structures are further optimized. The structural optimization of all geometries is carried out using the conjugate gradient method (Payne et al., 1992). The Brillouin zone is sampled by a $(2 \times 2 \times 1) \quad$ K-point grid using the Monkhorst-Pack scheme (Monkhorst and Pack, 1976). For density of states (DOS) calculations, Monkhorst and Pack generated a $(9 \times 9 \times 1)$ set of $\mathrm{K}$ points.

The ground state geometries of single transition-metal clusters ( $\mathrm{Mo}, \mathrm{Fe}$, and $\mathrm{V}$ ) are adsorbed on the abovementioned supports and the complexes optimized. The adsorption energy of $\mathrm{Mo}, \mathrm{V}$, and $\mathrm{Fe}$ on these supports is calculated as follows:

$$
\mathrm{E}_{\mathrm{ad}}=\mathrm{E}_{(\mathrm{M}-\text {-system })}-\mathrm{E}_{(\mathrm{M})}-\mathrm{E}_{(\text {system })},
$$

where $\mathrm{E}_{(\mathrm{M} \text {--system) }}$ represents the energy of the optimized single transition-metal cluster $(\mathrm{Mo}, \mathrm{Fe}$, and $\mathrm{V}$ ) and the designed surface supports. $\mathrm{E}_{(\mathrm{M})}$ and $\mathrm{E}_{(\text {system })}$ represent the energy of a single metal and surface support, respectively.

Finally, the $\mathrm{N}_{2}$ molecule is adsorbed on these active metal clusters $(\mathrm{Mo}, \mathrm{Fe}$, and $\mathrm{V})$ on graphene-based surface supports. A parallel mode of adsorption (both the nitrogen atoms are exposed to the metal) is used as this mode has been found to be more
TABLE 1 | Interatomic distances and adsorption energies of Mo, Fe, and $\mathrm{V}$ on various graphene-based supports (pristine graphene, defective graphene, $\mathrm{BN}$-doped graphene, BNC-ring graphene, BN-ring graphene, and adsorption energy are abbreviated as prisgp, dfgp, BNgp, BNCgp, BNrg_gp, and $\left.\mathrm{E}_{\mathrm{ad}}\right)$.

\begin{tabular}{|c|c|c|c|c|}
\hline System & C-metal (Å) & B-metal (Å) & N-metal (Å) & $E_{a d}(e V)$ \\
\hline prisgp_Mo & $2.200-2.213$ & - & - & -4.653 \\
\hline prisgp_Fe & $2.069-2.080$ & - & - & -2.602 \\
\hline prisgp_V & $2.147-2.170$ & - & - & -3.145 \\
\hline dfgp_Mo & $1.932-1.956$ & - & - & -10.893 \\
\hline dfgp_Fe & $1.766-1.768$ & - & - & -9.329 \\
\hline dfgp_V & $1.863-1.873$ & & & -9.744 \\
\hline BNgp_Mo & $2.145-2.261$ & 2.258 & 2.211 & -3.929 \\
\hline BNgp_Fe & $2.012-2.473$ & 2.303 & 1.861 & -1.090 \\
\hline BNgp_V & 2.079-2.192 & 2.215 & 2.172 & -2.494 \\
\hline BNCgp_Mo & $2.072-2.305$ & $2.279-2.28$ & $2.224-2.226$ & -3.864 \\
\hline BNCgp_Fe & $1.947-2.148$ & $2.106-2.218$ & $2.01-2.225$ & -1.728 \\
\hline BNCgp_V & $2.072-2.306$ & $2.279-2.28$ & $2.224-2.226$ & -2.498 \\
\hline BNrg_gp_Mo & - & $2.216-2.219$ & $2.267-2.273$ & -3.016 \\
\hline BNrg_gp_Fe & - & $2.063-2.124$ & $2.073-2.197$ & -1.048 \\
\hline BNrg_gp_V & - & $2.17-2.227$ & $2.204-2.226$ & -1.467 \\
\hline
\end{tabular}

effective as compared to the vertical mode. In the vertical mode, only one $\mathrm{N}$ atom in the $\mathrm{N}_{2}$ molecule interacts with the metal leading to weak activation (Song et al., 2021). 

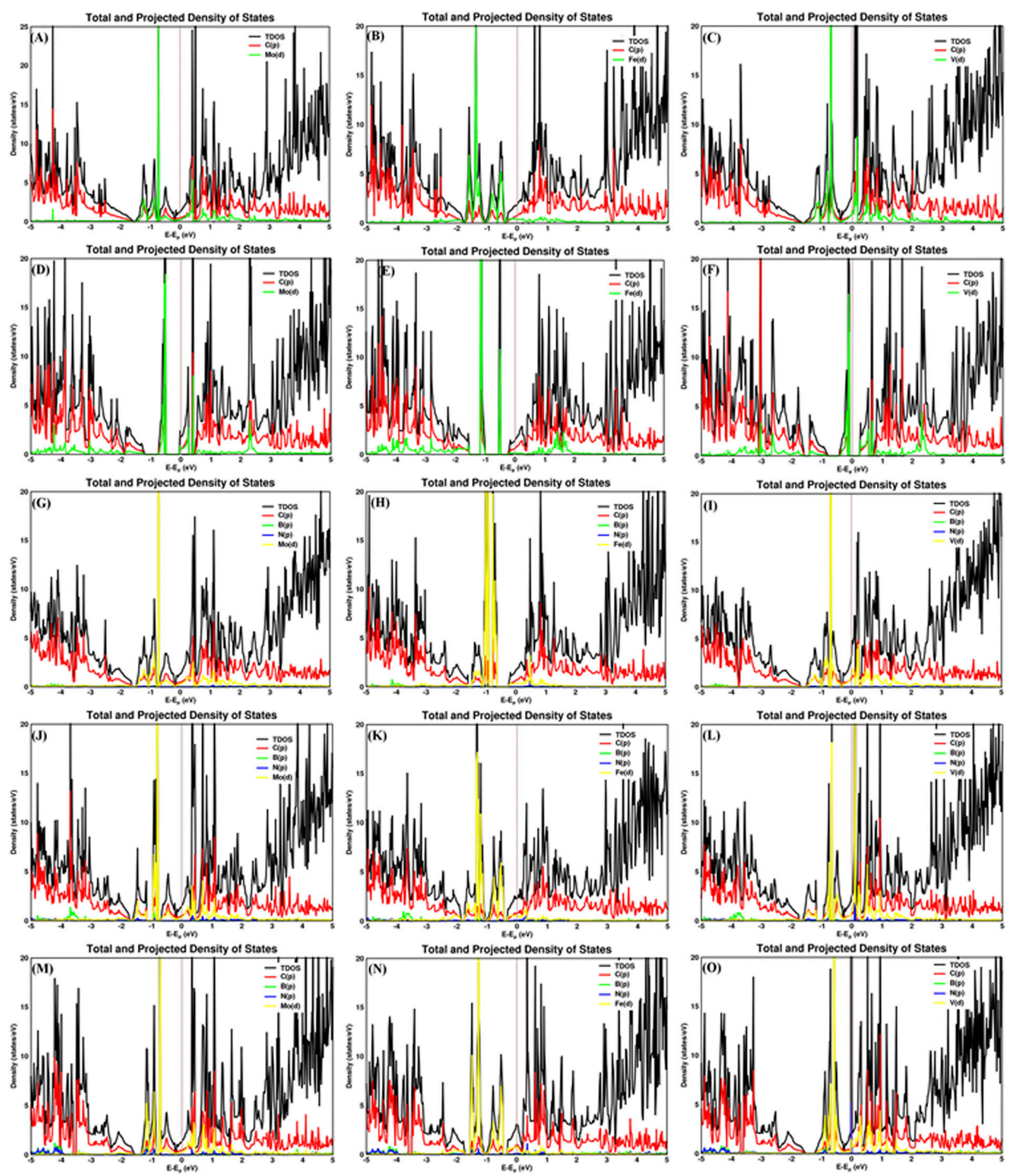

FIGURE 3 | Total and projected density of states of Mo, Fe, and V on (A-C) pristine graphene, (D-F) defective graphene, (G-I) BN-doped graphene, (J-L) BNCring graphene, and (M-O) BN-ring graphene.

The dissociated adsorption energy of the $\mathrm{N}_{2}$ molecule on the catalytic systems is calculated as follows:

$$
\mathrm{E}_{\mathrm{ad}}=\mathrm{E}_{(\mathrm{N} 2---\mathrm{M}-\text {-system })}-\mathrm{E}_{(\mathrm{N} 2)}-\mathrm{E}_{(\mathrm{M}-\text {-system })} \text {, }
$$

where $\mathrm{E}_{(\mathrm{N} 2---\mathrm{M}-\mathrm{-system})}$ represents the energy of the dissociated $\mathrm{N}_{2}$ molecule on the catalytic systems. $\mathrm{E}_{(\mathrm{N} 2)}$ and $\mathrm{E}_{(\mathrm{M} \text {--system) }}$ represent the energy of the $\mathrm{N}_{2}$ molecule and metal-adsorbed various surface supports, respectively. Nudged elastic band (NEB) calculations were performed toward prediction of energy barrier of $\mathrm{N}_{2}$ activation on metal-adsorbed $\mathrm{BN}$-doped graphene-based substrates. 


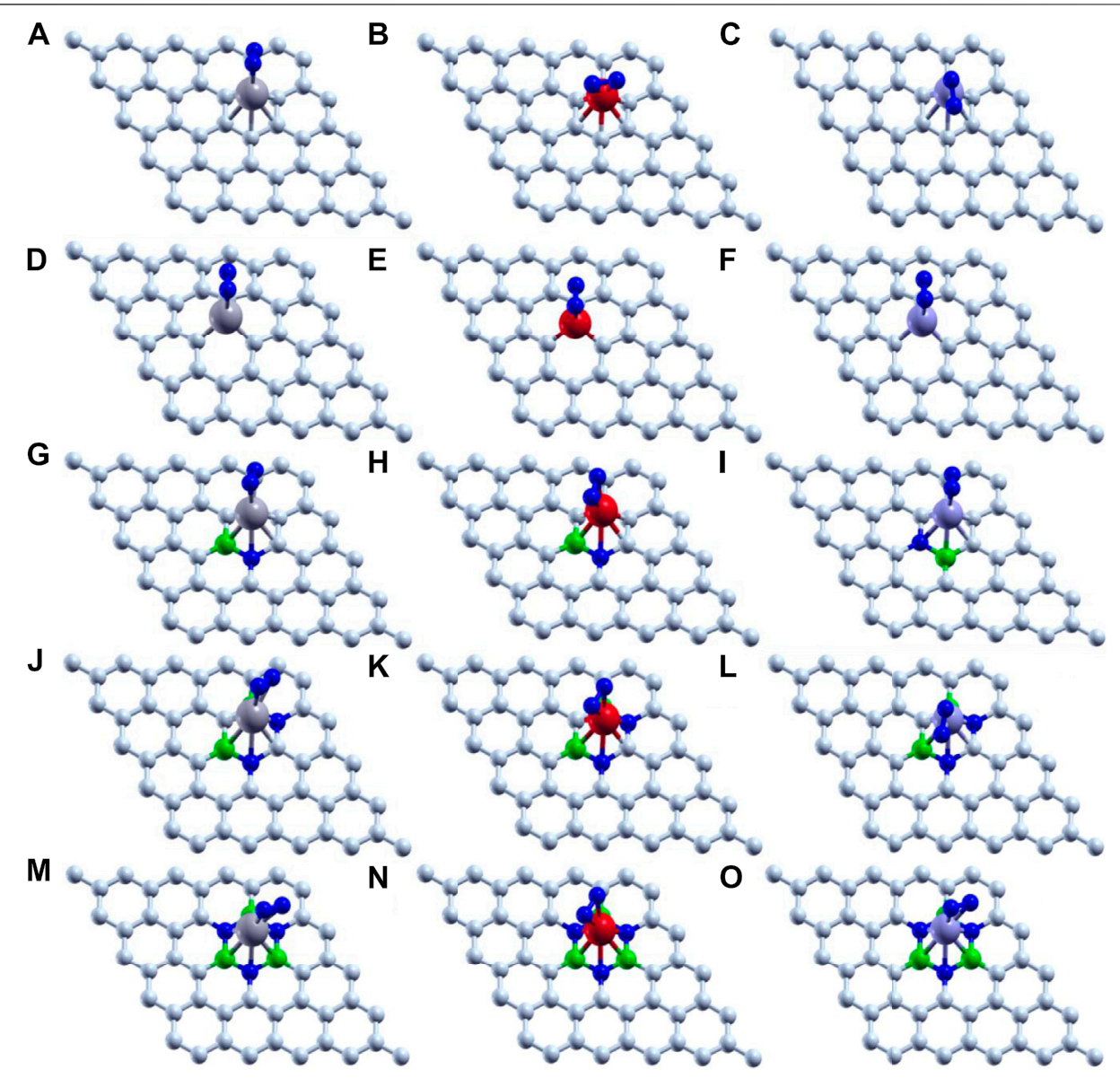

FIGURE 4 | Optimized structure of the $\mathrm{N}_{2}$ molecule adsorbed on $\mathrm{Mo}$ (gray), $\mathrm{Fe}$ (red), and $\mathrm{V}$ (purple) on various supports: (A-C) pristine graphene, (D-F) defective graphene, (G-I) BN-doped graphene, (J-L) BNC-ring graphene, and (M-O) BN-ring graphene.

\section{RESULTS AND DISCUSSION}

\section{Anchoring of Single Metal Atom (Mo, Fe, and V) on Various Graphene-Based Supports}

Graphene-based 2D materials which act as an electron reservoir are used as the support for adsorbing the single atom cluster (Mo, $\mathrm{Fe}$, and V) which increases the catalytic activity of the metal center. The five graphene-based supports are designed, viz., 1) pristine graphene (50 carbon atoms), 2) defective graphene (49 carbon atoms with a single vacancy at the center), 3) BN-doped graphene ( $4 \%$ heteroatom doping in which boron and nitrogen are substituted instead of carbon in the pristine graphene), 4) $\mathrm{BNC}$-ring graphene ( $8 \%$ heteroatom doping), and 5) $\mathrm{BN}$-ring graphene (12\% heteroatom doping). All these graphene-based supports are designed and optimized to the local minima as shown in Figure 1.

Thus, we have tried to establish the relative reactivity of single atom clusters (Mo, Fe, and V) chemisorbed on the abovementioned surfaces. The optimized structure of adsorption of
Mo (gray), Fe (red), and V (purple) on various surface supports is shown in Figure 2. The adsorption energy of a single metal atom (Mo, Fe, and V) on pristine graphene, defective graphene, BNdoped graphene, BNC-ring graphene, and BN-ring graphene is 4.653, 2.602, and 3.145 eV; 10.893, 9.329, and 9.744 eV; 3.929, 1.090, and 2.494 eV; 3.864, 1.728, and $2.498 \mathrm{eV}$; and 3.016, 1.048, and $1.467 \mathrm{eV}$, respectively. Comparatively, the adsorption energy of Mo on the designed supports is $\sim 2 \mathrm{eV}$ more due to its bulky nature with respect to other metals (Fe and V). Interestingly, the dangling carbon atoms at the center increase the adsorption energies for a defective graphene support better than the rest, and also the increase in the percentage of heteroatom (B and N) doping decreases the adsorption energies of the single metal atom on supports.

The carbon-metal (C-M) interatomic distance of $\mathrm{Mo}, \mathrm{Fe}$, and $\mathrm{V}$ on pristine graphene, defective graphene, $\mathrm{BN}$-doped graphene, and BNC-ring graphene is 2.200-2.213, 2.069-2.080, and

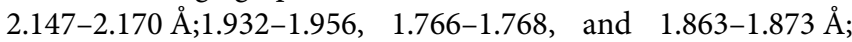
2.145-2.261, 2.012-2.473, and 2.079-2.192 Аं; and 2.072-2.305, 1.947-2.148, and 2.072-2.306 $\AA$, respectively. The boron-metal (B-M) interatomic distance of $\mathrm{Mo}, \mathrm{Fe}$, and $\mathrm{V}$ on $\mathrm{BN}$-doped 
TABLE 2 | Interatomic distances and adsorption energies of $\mathrm{N}_{2}$ on $\mathrm{Mo}$, V, and Fe on various graphene-based supports (pristine graphene, defective graphene, BN-doped graphene, BNC-ring graphene, BN-ring graphene, and adsorption energy are abbreviated as prisgp, dfgp, BNgp, BNCgp, BNrg_gp, and $\mathrm{E}_{\mathrm{ad}}$ ).

\begin{tabular}{|c|c|c|c|c|c|}
\hline System & C-metal (Å) & B-metal (Å) & $\mathbf{N}_{\text {doped }}-$ metal $(\AA ̊)$ & $\mathbf{N}_{\mathrm{ad}}$-metal (Å) & $E_{\text {ad }}(e V)$ \\
\hline prisgp_Mo & $2.228-2.313$ & - & - & $2.039-2.117$ & -1.739 \\
\hline prisgp_Fe & $2.077-2.162$ & - & - & $1.92-1.923$ & -1.334 \\
\hline prisgp_V & $2.190-2.264$ & - & - & $1.911-1.994$ & -1.996 \\
\hline dfgp_Mo & $1.946-2.013$ & - & - & 2.22-2.221 & -0.887 \\
\hline dfgp_Fe & $1.777-1.847$ & - & - & $1.964-2.078$ & -0.620 \\
\hline dfgp_V & $1.871-1.925$ & - & - & $2.161-2.218$ & -0.628 \\
\hline BNgp_Mo & $2.194-2.257$ & 2.313 & 2.32 & $2.027-2.091$ & -1.844 \\
\hline BNgp_Fe & $2.096-2.144$ & 2.18 & 2.202 & $1.902-1.907$ & -2.278 \\
\hline BNgp_V & $2.133-2.234$ & 2.319 & 2.223 & 1.918-2 & -1.988 \\
\hline BNCgp_Mo & $2.111-2.319$ & $2.316-2.407$ & $2.239-2.328$ & 2-2.057 & -1.870 \\
\hline BNCgp_Fe & $2.004-2.172$ & $2.219-2.222$ & $2.165-2.173$ & 1.889-1.89 & -1.544 \\
\hline BNCgp_V & $2.048-2.241$ & $2.303-2.342$ & 2.23-2.242 & $1.908-1.979$ & -2.116 \\
\hline BNrg_gp_Mo & - & $2.244-2.41$ & $2.274-2.341$ & 2-2.068 & -1.868 \\
\hline BNrg_gp_Fe & - & $2.141-2.216$ & $2.083-2.234$ & 1.9 & -1.510 \\
\hline BNrg_gp_V & - & $2.256-2.371$ & $2.264-2.297$ & $1.869-1.928$ & -2.258 \\
\hline
\end{tabular}

graphene, BNC-ring graphene, and $\mathrm{BN}$-ring graphene is 2.258, 2.303, and $2.215 \AA ; 2.279-2.28,2.106-2.218$, and 2.279-2.28 $\AA$; and $2.216-2.219,2.063-2.124$, and $2.17-2.227 \AA$, respectively. The nitrogen-metal (N-M) interatomic distance of Mo, Fe, and $\mathrm{V}$ on $\mathrm{BN}$-doped graphene, $\mathrm{BNC}$-ring graphene, and $\mathrm{BN}-$ ring graphene is 2.211, 1.861, and 2.172 $\AA$; 2.224-2.226, 2.01-2.225, and 2.224-2.226 $\AA$; and 2.267-2.273, 2.073-2.197, and 2.204-2.226 $\AA$, respectively. The interatomic distances and adsorption energies of Mo, Fe, and $\mathrm{V}$ on various graphene-based supports are shown in Table 1. Thus, the significance of the result shows that the adsorption energies of a single metal atom on the surface support provide a stable and potential catalyst for $\mathrm{N}_{2}$ activation. The total density of states and projected density of states of a single metal atom (Mo, Fe, and V) on graphene-based supports are shown in Figure 3. The total density of states (TDOS) and partial density of states (PDOS) reveal that the d-states of a single metal atom (Mo, Fe, and V) strongly hybridize with the p-state of unsaturated carbon atoms and heteroatoms (B and N). The d-state of a single metal atom shows its maximum density of states between -2 and $2 \mathrm{eV}$. On comparing, the p-state of unsaturated carbon atoms is maximum in pristine and defective supports which reveals that, in the other three supports, the p-state of both boron and nitrogen is hybridized with the d-state of metal.

\section{$\mathrm{N}_{2}$ Activation on Single Metal Atom (Mo, Fe, and V) Anchored on Various Graphene-Based Supports}

The adsorption energies of $\mathrm{N}_{2}$ on a single metal atom (Mo, Fe, and $\mathrm{V}$ ) on pristine graphene, defective graphene, BN-doped graphene, BNC-ring graphene, and $\mathrm{BN}$-ring graphene are $1.739,1.334$, and $1.996 \mathrm{eV} ; 0.887,0.620$, and $0.628 \mathrm{eV} ; 1.844$, 2.278, and $1.988 \mathrm{eV} ; 1.870,1.544$, and $2.116 \mathrm{eV}$; and 1.868, 1.510, and $2.258 \mathrm{eV}$, respectively. Comparatively, the adsorption energies of $\mathrm{N}_{2}$ on a single metal atom $(\mathrm{Mo}, \mathrm{Fe}$, and $\mathrm{V}$ ) on the defective graphene support are less compared to those on the rest of the support. Moreover, there is an eventual increase in adsorption energies of $\mathrm{N}_{2}$ on $\mathrm{V}$ on supports (BN-doped graphene, BNC-ring graphene, and BN-ring graphene) due to more vacant $d$ orbitals (less than half-filled), which is vice versa in Fe (more than half-filled $d$ orbitals) on the same supports. The optimized structure of adsorption of $\mathrm{N}_{2}$ on a single metal atom ( Mo, Fe, and V) on various surface supports is shown in Figure 4.

The carbon-metal $(\mathrm{C}-\mathrm{M})$ interatomic distance of $\mathrm{N}_{2}$ on a single metal atom ( $\mathrm{Mo}, \mathrm{Fe}$, and $\mathrm{V}$ ) on pristine graphene, defective graphene, BN-doped graphene, and BNC-ring graphene is $2.228-2.313,2.077-2.162$, and 2.190-2.264 Aं; 1.946-2.013, 1.777-1.847, and 1.871-1.925 $\AA$; 2.194-2.257, 2.096-2.144, and

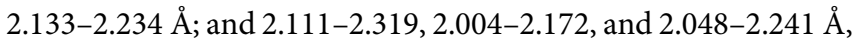
respectively. The boron-metal (B-M) interatomic distance of $\mathrm{N}_{2}$ on a single metal atom ( $\mathrm{Mo}, \mathrm{Fe}$, and $\mathrm{V}$ ) on $\mathrm{BN}$-doped graphene, BNC-ring graphene, and BN-ring graphene is 2.313, 2.18, and $2.319 \AA$; 2.316-2.407, 2.219-2.222, and 2.303-2.342 $\AA$; and $2.244-2.41,2.141-2.216$, and 2.256-2.371 $\AA$, respectively. The nitrogen-metal $\left(\mathrm{N}_{\text {doped }}-\mathrm{M}\right)$ interatomic distance of $\mathrm{N}_{2}$ on a single metal atom ( $\mathrm{Mo}, \mathrm{Fe}$, and $\mathrm{V}$ ) on $\mathrm{BN}$-doped graphene, $\mathrm{BNC}$-ring graphene, and $\mathrm{BN}$-ring graphene is 2.32, 2.202, and $2.223 \AA$; 2.239-2.328, 2.165-2.173, and 2.23-2.242 $\AA$; and 2.274-2.341, 2.083-2.234, and 2.264-2.297 A, respectively.

The nitrogen-metal $\left(\mathrm{N}_{\mathrm{ad}}-\mathrm{M}\right)$ interatomic distance of $\mathrm{N}_{2}$ on a single metal atom (Mo, Fe, and $\mathrm{V}$ ) on pristine graphene, defective graphene, BN-doped graphene, BNC-ring graphene, and BN-ring graphene is $2.039-2.117,1.92-1.923$, and 1.911-1.994 $\AA$; 2.22-2.221, 1.964-2.078, and 2.161-2.218 ; 2.027-2.091, 1.902-1.907, and

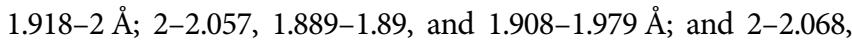
1.9 , and 1.869-1.928 $\AA$, respectively. The interatomic distances and adsorption energy of $\mathrm{N}_{2}$ on a single metal atom (Mo, Fe, and $\mathrm{V}$ ) on various substrate systems are shown in Table 2 . The total density of states and projected density of states of $\mathrm{N}_{2}$ on a single metal atom (Mo, Fe, and V) on the graphene-based support are shown in Figure 5. The total density of states (TDOS) and partial density of states (PDOS) reveal that the d-states of a single metal atom (Mo, $\mathrm{Fe}$, and $\mathrm{V}$ ) hybridize with the p-state of adsorbed nitrogen as well as carbon, boron, and nitrogen atoms doped on the support. Thus, the $\mathrm{d}$-state of a single metal atom shares its vacant orbital with the $\mathrm{p}$-state of hybridizing atoms. 


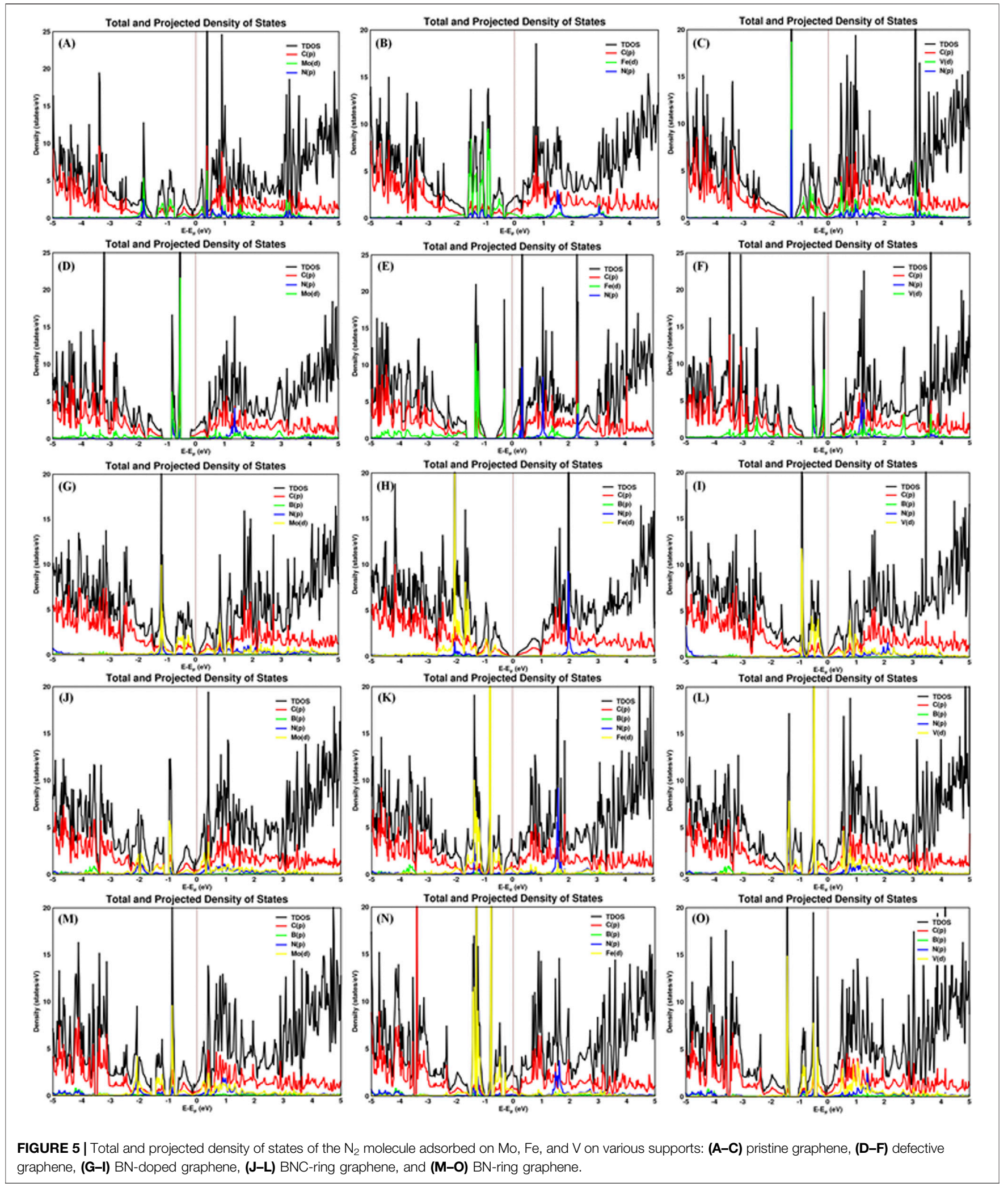


TABLE 3 | Structural, electronic, and vibrational properties of various catalytic systems for $\mathrm{N}_{2}$ activation (pristine graphene, defective graphene, BN-doped graphene, BNCring graphene, and BN-ring graphene are abbreviated as prisgp, dfgp, BNgp, BNCgp, and BNrg_gp).

\begin{tabular}{|c|c|c|c|c|}
\hline System & $\frac{\mathrm{N}-\mathrm{N} \text { bond length }}{(\AA)}$ & $\frac{\text { IR stretching }}{\left(\mathrm{cm}^{-1}\right)}$ & \multicolumn{2}{|c|}{ Charge on $\mathrm{N}_{2}(\mathrm{e})$} \\
\hline prisgp_Mo & 1.2 & 1735 & -0.3239 & -0.2274 \\
\hline prisgp_Fe & 1.18 & 1823 & -0.1556 & -0.3095 \\
\hline prisgp_V & 1.21 & 1,692 & -0.4328 & -0.1847 \\
\hline dfgp_Mo & 1.17 & 1907 & -0.3055 & -0.1237 \\
\hline dfgp_Fe & 1.16 & 2009 & -0.1954 & -0.1228 \\
\hline dfgp_V & 1.16 & 1997 & -0.1359 & -0.1935 \\
\hline BNgp_Mo & 1.2 & 1701 & -0.2088 & -0.363 \\
\hline BNgp_Fe & 1.19 & 1802 & -0.2152 & -0.252 \\
\hline BNgp_V & 1.2 & 1711 & -0.2733 & -0.3433 \\
\hline BNCgp_Mo & 1.21 & 1,636 & -0.2783 & -0.3213 \\
\hline BNCgp_Fe & 1.19 & 1777 & -0.2714 & -0.2093 \\
\hline BNCgp_V & 1.21 & 1,688 & -0.2711 & -0.352 \\
\hline BNrg_gp_Mo & 1.21 & 1,666 & -0.2584 & -0.3194 \\
\hline BNrg_gp_Fe & 1.19 & 1796 & -0.314 & -0.1728 \\
\hline BNrg_gp_V & 1.23 & 1,597 & -0.2666 & -0.4262 \\
\hline
\end{tabular}

\section{N-N Bond Stretching Frequency Analysis on Designed Catalytic Systems}

To probe the stretching frequency of the adsorbed $\mathrm{N}_{2}$ molecule on a single metal atom (Mo, $\mathrm{Fe}$, and $\mathrm{V}$ ) on the graphene-based support, we investigated the spectral range of $1,300-2,300 \mathrm{~cm}^{-1}$, which covers the typical frequencies of the different $\mathrm{N}_{2}$ species known to exist on transition-metal surfaces. The stretching frequency of the unbound $\mathrm{N}_{2}$ molecule is attributed to $2,330 \mathrm{~cm}^{-1}$, and the $\mathrm{N}-\mathrm{N}$ bond length is $1.09 \AA$ (Shi and Jacobi, 1992). The $\mathrm{N}-\mathrm{N}$ bond length and IR stretching frequency $v(\mathrm{~N}-\mathrm{N})$ of $\mathrm{N}_{2}$ on a single metal atom $(\mathrm{Mo}, \mathrm{Fe}$, and $\mathrm{V})$ on pristine graphene, defective graphene, BN-doped graphene, $\mathrm{BNC}$-ring graphene, and $\mathrm{BN}$-ring graphene are $1.20 \AA \quad\left(1735 \mathrm{~cm}^{-1}\right), \quad 1.18 \AA \quad\left(1823 \mathrm{~cm}^{-1}\right), \quad$ and $1.21 \AA$ $\left(1,692 \mathrm{~cm}^{-1}\right) ; 1.17 \AA\left(1907 \mathrm{~cm}^{-1}\right), \quad 1.16 \AA\left(2009 \mathrm{~cm}^{-1}\right)$, and $1.16 \AA\left(1997 \mathrm{~cm}^{-1}\right) ; 1.20 \AA\left(1701 \mathrm{~cm}^{-1}\right), 1.19 \AA\left(1802 \mathrm{~cm}^{-1}\right)$, and $\quad 1.20 \AA \quad\left(1711 \mathrm{~cm}^{-1}\right) ; \quad 1.21 \AA \quad\left(1,636 \mathrm{~cm}^{-1}\right), \quad 1.19 \AA$ $\left(1777 \mathrm{~cm}^{-1}\right)$, and $1.21 \AA\left(1,688 \mathrm{~cm}^{-1}\right)$; and $1.21 \AA\left(1,666 \mathrm{~cm}^{-1}\right)$, $1.19 \AA\left(1796 \mathrm{~cm}^{-1}\right)$, and $1.23 \AA\left(1,597 \mathrm{~cm}^{-1}\right)$, respectively.

The Bader charge analysis (Bader, 1991; Tang et al., 2009) clearly demonstrates the charge redistribution between the activated nitrogen atoms and the active metal centered on support-based catalysts. The structural, electronic, and vibrational properties of various catalytic systems for $\mathrm{N}_{2}$ activation are listed in Table 3. The N-N stretching frequency, $\mathrm{N}-\mathrm{N}$ bond length, and charge on nitrogen of the $\mathrm{N}_{2}$ molecule adsorbed on $\mathrm{Mo}, \mathrm{Fe}$, and $\mathrm{V}$ on various graphene supports are shown in Supplementary Figure S1.

As a case study, the potential of the Mo-adsorbed BN-doped graphene catalyst for the activation of $\mathrm{N}_{2}$ is discussed in Supplementary Figure S2. NEB calculation is performed in between these reactants and products to confirm the $\mathrm{N}_{2}$ activation energy barrier. Mo-adsorbed $\mathrm{BN}$-doped graphene and gaseous nitrogen are considered reactants. Thus, the Mo-adsorbed BNdoped graphene catalyst shows more feasible $\mathrm{N}_{2}$ activation with an effective energy barrier of $3.21 \mathrm{eV}$. The activation barrier plot of the $\mathrm{N}_{2}$ molecule adsorbed on Mo on the BN-doped graphene support is shown in Supplementary Figure S2 (Liu et al., 2021).

\section{CONCLUSION}

In this work, we explore the potential of various graphene-based $2 \mathrm{D}$ materials, viz., pristine, defective, $\mathrm{BN}$-doped graphene, etc., as a support for a single atom cluster ( $\mathrm{Mo}, \mathrm{Fe}$, and $\mathrm{V})$. These graphene-based supports show excellent potential toward the anchoring of a single atom cluster (Mo, $\mathrm{Fe}$, and $\mathrm{V}$ ) with adsorption energies ranging between 1.048 and $10.893 \mathrm{eV}$. Thus, the adsorption energies vary substantially with respect to the graphene-based supports, viz., pristine, defective, BN doped, etc. This is attributed to the size and nature of hybridization between the $d$ orbitals of the interacting single metal atom ( $\mathrm{Mo}, \mathrm{Fe}$, and $\mathrm{V}$ ) and the $s p^{2}$ orbitals of unsaturated carbon atoms of various designed graphene-based supports. The catalytic performance of a single metal atom $(\mathrm{Mo}, \mathrm{Fe}$, and $\mathrm{V}$ ) on graphene-supported catalysts is explored for the activation of molecular nitrogen. The adsorption energies of the nitrogen molecule on a graphene-supported single atom cluster ( $\mathrm{Mo}, \mathrm{Fe}$, and $\mathrm{V}$ ) range between 0.620 and $2.278 \mathrm{eV}$, which is attributed to the interacting environment of the active metal centered on the support and the $p$ orbital of adsorbed molecular nitrogen. Bader charge and density of states analyses corroborate an enhanced hybridization between the $d$ states of the single metal atoms (Mo, Fe, and $\mathrm{V})$ and adsorbed molecular nitrogen for activation. The N-N stretching frequencies are found which are considerably redshifted ranging from $2009 \mathrm{~cm}^{-1}(1.16 \AA)$ to $1,597 \mathrm{~cm}^{-1}$ $\left(1.23 \AA\right.$ ) compared to that of the unbound $\mathrm{N}_{2}$ molecule $\left(2,330 \mathrm{~cm}^{-1}(1.09 \AA)\right)$. Thus, from the results, we understood that even a single metal atom (Mo, Fe, and V) with functionalized (BN-doped) graphene supports can highlight the excellent potential for nitrogen activation. 


\section{DATA AVAILABILITY STATEMENT}

The original contributions presented in the study are included in the article/Supplementary Material, and further inquiries can be directed to the corresponding authors.

\section{AUTHOR CONTRIBUTIONS}

SaK conceptualized the research idea. TS investigated the data and wrote the original draft. TS, SeK, and SaK were involved in formal analysis. TS and SaK validated the results and reviewed and edited the paper. SeK and SaK supervised the work. SeK was involved in funding acquisition and project administration.

\section{FUNDING}

DST-SERB funded the N-PDF (National Post-Doctoral Fellowship) (File Number: PDF/2016/002785).

\section{REFERENCES}

Bader, R. F. W. (1991). A Quantum Theory of Molecular Structure and its Applications. Chem. Rev. 91, 893-928. doi:10.1021/cr00005a013

Blöchl, P. E. (1994). Projector Augmented-Wave Method. Phys. Rev. B 50, 17953-17979. doi:10.1103/physrevb.50.17953

Cao, B., Starace, A. K., Judd, O. H., Bhattacharyya, I., Jarrold, M. F., López, J. M., et al. (2010). Activation of Dinitrogen by Solid and Liquid Aluminum Nanoclusters: a Combined Experimental and Theoretical Study. J. Am. Chem. Soc. 132, 12906-12918. doi:10.1021/ja103356r

Cao, B., Starace, A. K., Judd, O. H., Bhattacharyya, I., and Jarrold, M. F. (2009). Metal Clusters with Hidden Ground States: Melting and Structural Transitions in Al115+, Al116+, and Al117+. J. Chem. Phys. 131, 124305. doi:10.1063/1.3224124

Chen, C., Kang, Y., Huo, Z., Zhu, Z., Huang, W., Xin, H. L., et al. (2014). Highly Crystalline Multimetallic Nanoframes with Three-Dimensional Electrocatalytic Surfaces. Science 343, 1339-1343. doi:10.1126/science.1249061

Cherkasov, N., Ibhadon, A. O., and Fitzpatrick, P. (2015). A Review of the Existing and Alternative Methods for Greener Nitrogen Fixation. Chem. Eng. Process. Process Intensification 90, 24-33. doi:10.1016/j.cep.2015.02.004

Choi, W. I., Wood, B. C., Schwegler, E., and Ogitsu, T. (2015). Combinatorial Search for High-Activity Hydrogen Catalysts Based on Transition-Metal-Embedded Graphitic Carbons. Adv. Energ. Mater. 5, 1501423. doi:10.1002/aenm.201501423

Dance, I. (2008). The Chemical Mechanism of Nitrogenase: Calculated Details of the Intramolecular Mechanism for Hydrogenation of $\eta 2-\mathrm{N} 2$ on FeMo-Co to NH3. Dalton Trans. 43, 5977-5991. doi:10.1039/b806100a

Das, S., Pal, S., and Krishnamurty, S. (2014). Dinitrogen Activation by Silicon and Phosphorus Doped Aluminum Clusters. J. Phys. Chem. C 118, 19869-19878. doi:10.1021/jp505700a

Einsle, O., Tezcan, F. A., Andrade, S. L., Schmid, B., Yoshida, M., Howard, J. B., et al. (2002). Nitrogenase MoFe-Protein at 1.16 A Resolution: A Central Ligand in the FeMo-Cofactor. Science 297, 1696-1700. doi:10.1126/science.1073877

Fajardo, J., Jr, and Peters, J. C. (2017). Catalytic Nitrogen-To-Ammonia Conversion by Osmium and Ruthenium Complexes. J. Am. Chem. Soc. 139, 16105-16108. doi:10.1021/jacs.7b10204

Fei, H., Dong, J., Feng, Y., Allen, C. S., Wan, C., Volosskiy, B., et al. (2018). General Synthesis and Definitive Structural Identification of MN4C4 Single-Atom Catalysts with Tunable Electrocatalytic Activities. Nat. Catal. 1, 63-72. doi:10.1038/s41929-017-0008-y

Fryzuk, M. D., and Johnson, S. A. (2000). The Continuing story of Dinitrogen Activation. Coord. Chem. Rev. 200-202, 379-409. doi:10.1016/s0010-8545(00) 00264-2

\section{ACKNOWLEDGMENTS}

TS acknowledges DST-SERB for funding the N-PDF (National Post-Doctoral Fellowship) and Pragnya for supporting with activation barrier calculations. SK and SK both acknowledge the High-Performance Computing facility provided by CSIR-NCL, Pune, and CSIR-4PI, Bangalore. The authors dedicate this article to Sourav $\mathrm{Pal}$ for his landmark contributions in the area of computational chemistry in catalysis. He is an excellent teacher and a wonderful guide who has inspired many generations of students.

\section{SUPPLEMENTARY MATERIAL}

The Supplementary Material for this article can be found online at: https://www.frontiersin.org/articles/10.3389/fchem.2021.733422/ full\#supplementary-material

Guo, X., Liu, S., and Huang, S. (2018). Single Ru Atom Supported on Defective Graphene for Water Splitting: DFT and Microkinetic Investigation. Int. J. Hydrogen Energ. 43, 4880-4892. doi:10.1016/j.ijhydene.2018.01.122

Jeon, I. Y., Choi, H. J., Ju, M. J., Choi, I. T., Lim, K., Ko, J., et al. (2013). Direct Nitrogen Fixation at the Edges of Graphene Nanoplatelets as Efficient Electrocatalysts for Energy Conversion. Sci. Rep. 3, 2260-2267. doi:10.1038/ srep02260

Kerpal, C., Harding, D. J., Lyon, J. T., Meijer, G., and Fielicke, A. (2013). N2 Activation by Neutral Ruthenium Clusters. J. Phys. Chem. C 117, 12153-12158. doi:10.1021/jp401876b

Kim, J., and Rees, D. (1992). Structural Models for the Metal Centers in the Nitrogenase Molybdenum-Iron Protein. Science 257, 1677-1682. doi:10.1126/ science. 1529354

Kresse, G., and Furthmüller, J. (1996). Efficiency of Ab-Initio Total Energy Calculations for Metals and Semiconductors Using a Plane-Wave Basis Set. Comput. Mater. Sci. 6, 15-50. doi:10.1016/0927-0256(96)00008-0

Kulkarni, B. S., Krishnamurty, S., and Pal, S. (2011). Size- and Shape-Sensitive Reactivity Behavior of Aln $(\mathrm{N}=2-5,13,30$, and 100) Clusters toward the N2 Molecule: A First-Principles Investigation. J. Phys. Chem. C 115, 14615-14623. doi:10.1021/jp203452a

Kumar, C. V. S., and Subramanian, V. (2017). Can boron Antisites of BNNTs Be an Efficient Metal-free Catalyst for Nitrogen Fixation? - A DFT Investigation. Phys. Chem. Chem. Phys. 19, 15377-15387. doi:10.1039/c7cp02220d

Kumar, D., Pal, S., and Krishnamurty, S. (2016). N2 Activation on Al Metal Clusters: Catalyzing Role of BN-Doped Graphene Support. Phys. Chem. Chem. Phys. 18, 27721-27727. doi:10.1039/c6cp03342c

Le, Y.-Q., Gu, J., and Tian, W. Q. (2014). Nitrogen-fixation Catalyst Based on Graphene: Every Part Counts. Chem. Commun. 50, 13319-13322. doi:10.1039/ c4cc01950d

Légaré, M.-A., Bélanger-Chabot, G., Dewhurst, R. D., Welz, E., Krummenacher, I., Engels, B., et al. (2018). Nitrogen Fixation and Reduction at boron. Science 359, 896-900. doi:10.1126/science.aaq1684

Li, H., Shang, J., Ai, Z., and Zhang, L. (2015). Efficient Visible Light Nitrogen Fixation with BiOBr Nanosheets of Oxygen Vacancies on the Exposed $\{001\}$ Facets. J. Am. Chem. Soc. 137, 6393-6399. doi:10.1021/jacs.5b03105

Li, X.-F., Li, Q.-K., Cheng, J., Liu, L., Yan, Q., Wu, Y., et al. (2016). Conversion of Dinitrogen to Ammonia by FeN3-Embedded Graphene. J. Am. Chem. Soc. 138, 8706-8709. doi:10.1021/jacs.6b04778

Liu, B., Manavi, N., Deng, H., Huang, C., Shan, N., Chikan, V., et al. (2021). Activation of N2 on Manganese Nitride-Supported Ni3 and Fe3 Clusters and Relevance to Ammonia Formation. J. Phys. Chem. Lett. 12, 6535-6542. doi:10.1021/acs.jpclett.1c01752 
Liu, J.-C., Tang, Y., Wang, Y.-G., Zhang, T., and Li, J. (2018). Theoretical Understanding of the Stability of Single-Atom Catalysts. Natl. Sci. Rev. 5, 638-641. doi:10.1093/nsr/nwy094

Lu, Y., Yang, Y., Zhang, T., Ge, Z., Chang, H., Xiao, P., et al. (2016). Photoprompted Hot Electrons from Bulk Cross-Linked Graphene Materials and Their Efficient Catalysis for Atmospheric Ammonia Synthesis. ACS nano 10, 10507-10515. doi:10.1021/acsnano.6b06472

Maibam, A., Govindaraja, T., Selvaraj, K., and Krishnamurty, S. (2019). Dinitrogen Activation on Graphene Anchored Single Atom Catalysts: Local Site Activity or Surface Phenomena. J. Phys. Chem. C 123, 27492-27500. doi:10.1021/acs.jpcc.9b06757

Maibam, A., and Krishnamurty, S. (2021). Nitrogen Activation to Reduction on a Recyclable V-SAC/BN-graphene Heterocatalyst Sifted through Dual and Multiphilic Descriptors. J. Colloid Interf. Sci. 600, 480-491. doi:10.1016/ j.jcis.2021.05.027

Monkhorst, H. J., and Pack, J. D. (1976). Special Points for Brillouin-Zone Integrations. Phys. Rev. B 13, 5188-5192. doi:10.1103/physrevb.13.5188

Ouyang, Y., Li, Q., Shi, L., Ling, C., and Wang, J. (2018). Molybdenum Sulfide Clusters Immobilized on Defective Graphene: a Stable Catalyst for the Hydrogen Evolution Reaction. J. Mater. Chem. A. 6, 2289-2294. doi:10.1039/c7ta09828f

Payne, M. C., Teter, M. P., Allan, D. C., Arias, T. A., and Joannopoulos, J. D. (1992). Iterative Minimization Techniques Forab Initiototal-Energy Calculations: Molecular Dynamics and Conjugate Gradients. Rev. Mod. Phys. 64, 1045-1097. doi:10.1103/revmodphys.64.1045

Perdew, J. P., Burke, K., and Ernzerhof, M. (1996). Generalized Gradient Approximation Made Simple. Phys. Rev. Lett. 77, 3865-3868. doi:10.1103/ physrevlett.77.3865

Qiao, B., Wang, A., Yang, X., Allard, L. F., Jiang, Z., Cui, Y., et al. (2011). Singleatom Catalysis of CO Oxidation Using Pt1/FeOx. Nat. Chem 3, 634-641. doi:10.1038/nchem.1095

Roy, D., Navarro-Vazquez, A., and Schleyer, P. v. R. (2009). Modeling Dinitrogen Activation by Lithium: A Mechanistic Investigation of the Cleavage of N2 by Stepwise Insertion into Small Lithium Clusters. J. Am. Chem. Soc. 131, 13045-13053. doi:10.1021/ja902980j

Seh, Z. W., Kibsgaard, J., Dickens, C. F., Chorkendorff, I., Nørskov, J. K., and Jaramillo, T. F. (2017). Combining Theory and experiment in Electrocatalysis: Insights into Materials Design. Science 355, eaad4998. doi:10.1126/science.aad4998

Sellmann, D., and Sutter, J. (1997). In Quest of Competitive Catalysts for Nitrogenases and Other Metal Sulfur Enzymes. Acc. Chem. Res. 30, 460-469. doi:10.1021/ar960158h

Shi, H., and Jacobi, K. (1992). Evidence for Physisorbed N2 in the Monolayer on $\mathrm{Ru}(001)$ at 40 K. Surf. Sci. 278, 281-285. doi:10.1016/0039-6028(92)90664-r

Song, R., Yang, J., Wang, M., Shi, Z., Zhu, X., Zhang, X., et al. (2021). Theoretical Study on P-Coordinated Metal Atoms Embedded in Arsenene for the
Conversion of Nitrogen to Ammonia. ACS omega 6, 8662-8671. doi:10.1021/acsomega.1c00581

Stüeken, E. E., Buick, R., Guy, B. M., and Koehler, M. C. (2015). Isotopic Evidence for Biological Nitrogen Fixation by Molybdenum-Nitrogenase from 3.2 Gyr. Nature 520, 666-669. doi:10.1038/nature14180

Tanabe, Y., and Nishibayashi, Y. (2016). Catalytic Dinitrogen Fixation to Form Ammonia at Ambient Reaction Conditions Using Transition Metal-Dinitrogen Complexes. Chem. Rec. 16, 1549-1577. doi:10.1002/tcr.201600025

Tang, W., Sanville, E., and Henkelman, G. (2009). A Grid-Based Bader Analysis Algorithm without Lattice Bias. J. Phys. Condens. Matter 21, 084204. doi:10.1088/0953-8984/21/8/084204

Wang, A., Li, J., and Zhang, T. (2018). Heterogeneous Single-Atom Catalysis. Nat. Rev. Chem. 2, 65-81. doi:10.1038/s41570-018-0010-1

Yan, H., Zhao, X., Guo, N., Lyu, Z., Du, Y., Xi, S., et al. (2018). Atomic Engineering of High-Density Isolated Co Atoms on Graphene with Proximal-Atom Controlled Reaction Selectivity. Nat. Commun. 9, 3197-3199. doi:10.1038/ s41467-018-05754-9

Yan, X., Liu, D., Cao, H., Hou, F., Liang, J., and Dou, S. X. (2019). Nitrogen Reduction to Ammonia on Atomic-Scale Active Sites under Mild Conditions. Small Methods 3, 1800501. doi:10.1002/smtd.201800501

Yang, X.-F., Wang, A., Qiao, B., Li, J., Liu, J., and Zhang, T. (2013). Single-Atom Catalysts: A New Frontier in Heterogeneous Catalysis. Acc. Chem. Res. 46, 1740-1748. doi:10.1021/ar300361m

Zhao, J., and Chen, Z. (2017). Single Mo Atom Supported on Defective boron Nitride Monolayer as an Efficient Electrocatalyst for Nitrogen Fixation: a Computational Study. J. Am. Chem. Soc. 139, 12480-12487. doi:10.1021/jacs.7b05213

Conflict of Interest: The authors declare that the research was conducted in the absence of any commercial or financial relationships that could be construed as a potential conflict of interest.

Publisher's Note: All claims expressed in this article are solely those of the authors and do not necessarily represent those of their affiliated organizations, or those of the publisher, the editors and the reviewers. Any product that may be evaluated in this article, or claim that may be made by its manufacturer, is not guaranteed or endorsed by the publisher.

Copyright $@ 2021$ Senthamaraikannan, Kaliaperumal and Krishnamurty. This is an open-access article distributed under the terms of the Creative Commons Attribution License (CC BY). The use, distribution or reproduction in other forums is permitted, provided the original author(s) and the copyright owner(s) are credited and that the original publication in this journal is cited, in accordance with accepted academic practice. No use, distribution or reproduction is permitted which does not comply with these terms. 